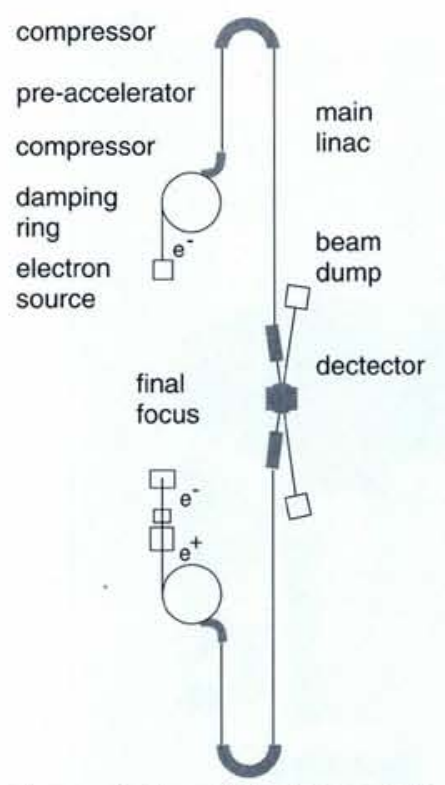

A generic layout of a linear collider for particle physics.

\title{
Research and Development for Linear Colliders
}

Owing to the inherent simplicity of the annihilation process, the study of electron-positron interactions has yielded a wealth of information about the basic constituents of matter and the forces which act between them. The incoming electrons and positrons annihilate to form a time-like electroweak current with well-defined quantum numbers which in turn couples directly to the basic constituents of matter. Thus, the cross-sections are well-known and the annihilation process leads to a well-defined finalstate topology. New particles can therefore be easily found and their properties unambiguously determined in a clean environment. Conversely, a negative result in a search can be used to define mass-limits on new particles. Generally speaking, therefore, it is desirable to extend $e^{+} e^{-}$experiments beyond the energies avaliable at CERN's LEP collider. Indeed, an $\mathrm{e}^{+} \mathrm{e}^{-}$collider which covers the mass range between the $\mathrm{W}^{+} \mathrm{W}^{-}$pair-production threshold and $500 \mathrm{GeV}$, with luminosities in excess of $10^{33} \mathrm{~cm}^{-2} \mathrm{~s}^{-1}$ will have a rich physics programme complementary to the programme at large hadron colliders.
It has become apparent that $\mathrm{e}^{+} \mathrm{e}^{-}$storage rings have reached with LEP 200 (the upgraded LEP machine) their maximum economic size. Because of the rapidly increasing synchrotron radiation losses in storage rings at energies above $200 \mathrm{GeV}$, linear $\mathrm{e}^{+} \mathrm{e}^{-}$colliders seem to be the better way to produce $\mathrm{e}^{+} \mathrm{e}^{-}$collisions at centre-of-mass energies higher than $200 \mathrm{GeV}$. They represent a new type of highenergy physics machine and the first one to be contemplated - a linear collider for a centre ofmass energy of $500 \mathrm{GeV}$ - is already a huge commitment in turms of money and manpower. For this reason, there is an intense world-wide research effort into the physical and technological aspects of linear colliders. Studies are in progress in many laboratories and DESY, in collaboration with many other laboratories, is investigating in parallel two realistic approaches for a 500-2000 GeV linear collider. The aim is to make a rational decision on the preferred approach in 1997.

There is general agreement that a low-frequency $(1300 \mathrm{MHz})$ superconducting linear accelerator

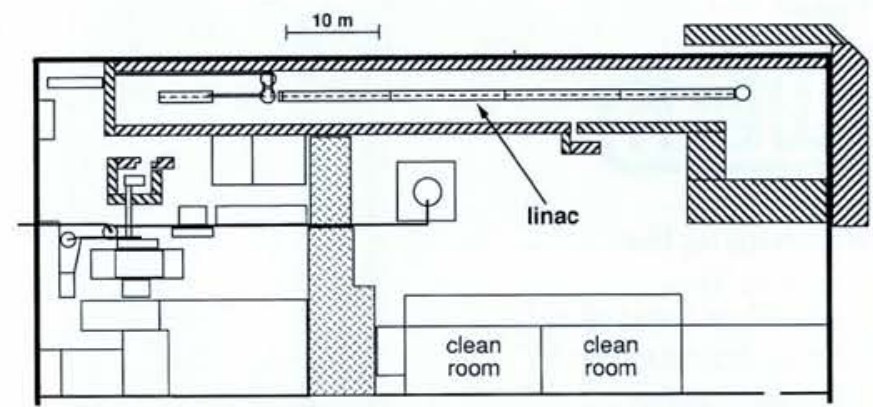

A plan of TESLA Test Facility at DESY.

The development of new accelerator technologies is an integral part of the research programme at DESY. The picture shows a nine-cell niobium cavity being prepared for chemical treatment. The development of superconducting cavities of high gradient is done in the framework of the international TESLA collaboration to prepare for the TESLA Test Facility.

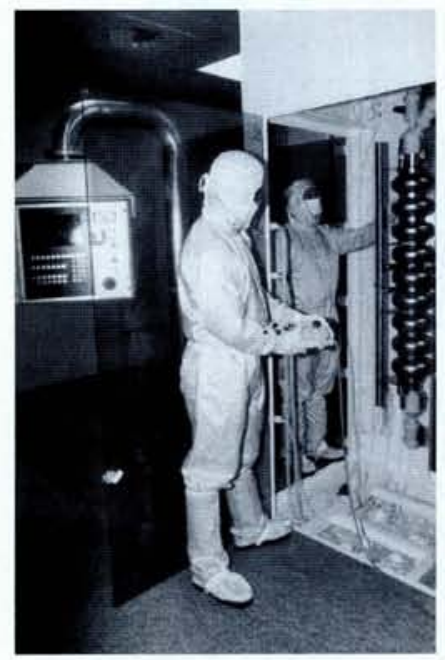

\section{The TESLA Approach}

The maximum accelerating gradient which can be reached in a well-designed superconducting niobium cavity is limited to values of around $50 \mathrm{mVv} / \mathrm{m}$ by the maximum strength of the magnetic field at the cavity surface. At present, the gradient is limited to much lower values by field emission from localized regions on the surface. An analysis shows that emission is not due to any inherent properties of the niobium, but is instead caused by specs of foreign materials attached to the cavity surface. A combination of stringent measures to keep the cavity surface clean and methods to remove any emitters that remain are therefore needed in order to reproducibly reach gradients above $20 \mathrm{mV} / \mathrm{m}$. Indeed, multicell cavities made of highpurity niobium materials, chemically polished and processed at high temperatures in a low pressure $\mathrm{Ti}$ atmosphere $\left(1500^{\circ} \mathrm{C}, 10^{-7}\right.$ torr $)$ or subjected to an RF treatment at a high peak power, consistently reach accelerating gradients of $15-20 \mathrm{mV} / \mathrm{m}$.

Very encouraging results on 5 -cell $1.3 \mathrm{GHz}$ cavities designed for the TESLA machine have been obtained in a collaboration between Cornell, DESY and Fermilab, using existing facilities at Cornell. Three cavities, made of high-purity solid niobium, were chemically polished and then subjected to the high peak-power treatment. They reached the design gradient of $25 \mathrm{mV} / \mathrm{m}$ or higher at the design impedance value of $3 \times 10^{9}$

The TESLA Collaboration, which at present consists of scientists from IHEP Beijing, TU Berlin, CERN, Cornell, TH Darmstadt, DESY, Fermilab, the University of Frankfurt/Main, INFN Frascati, INFN Milan, IPN Orsay, KFK Karlsruhe, the University of Karlsruhe, LAL Orsay, CEA Saclay, SEFT Helsinki, UCLA, and the University of Wuppertal, has constructed a large facility at DESY to process and test niobium cavities. The processing section, which includes a high-purity chemical polishing facility, clean rooms, a high-temperature, high-vacuum furnace, a $1.5 \mathrm{~K}$ cryogenic plant feeding vertical and horizontal test cryostats, and a $4.5 \mathrm{MW}, 1.3 \mathrm{GHz}$ high peak-power treatment installation, is in the process of being commissioned. A total of fourteen $1.3 \mathrm{GHz}$, 9-cell cavities made from high-purity solid niobium are on order. Two have already been delivered, and the first test results are expected later this summer.

The next step is to reduce the cost per metre of the active cavity. To this end, eight $1.03 \mathrm{~m}$ niobium cavities will be installed in a single $12 \mathrm{~m}$ long, low thermal loss cryostat. The number of auxiliary components such as higher order mode absorbers, power couplers, tuners, vacuum insulation valves, etc. has been reduced considerably compared to earlier designs in order to reduce costs. The helium transfer lines are incorporated into the cryostat.

The first prototype cryostat of the new design is presently being constructed in Italy and the delivery is scheduled for late-1994. The expected static heat loss is less than $0.5 \mathrm{~W} / \mathrm{m}$ at $1.8 \mathrm{~K}$, a factor of 10 improvement over present designs. Components such as tuners, higher order mode couplers and power couplers have been designed and in part constructed and tested. Superconducting focusing quadrupoles, beam-position monitors and steering magnets will be mounted at the end of each cryomodule (the design of these components is well advanced). The first $12 \mathrm{~m}$ long cryomodule, including all the auxiliaries, is expected to be ready for cool-down in the autumn of 1995.

As an integrated system test, the TESLA group is proposing to accelerate an electron beam through a string of four of the cryomodules. The $15 \mathrm{MV}$ injector for the project is presently being designed and built in a collaborative effort between laboratories at Orsay and Saclay. One $4.5 \mathrm{MW}$ peak power klystron with RF pulse length of $1.5 \mathrm{~ms}$ will feed 16 cavities in two cryomodules. Two klystrons have been delivered and the first modulator, designed and constructed at Fermilab is available. Operation of the $500 \mathrm{MeV}$ superconducting linac, which is scheduled for 1997, will be used to demonstrate the technical feasibility of the TESLA approach and provide the basis for a firm cost-estimate. 
would be the best approach, from almost every perspective, for a linear collider. The main problem, however, is the costs of such a machine. Only superconducting linacs with accelerating gradients of $25 \mathrm{mV} / \mathrm{m}$ (a factor of 5-times the gradient in systems operating today), built at a cost per metre of less than $25 \%$ of present systems, can compete on a cost basis with other approaches for a linear collider. Recent advances in superconducting radio-frequency technology make it appear that such a goal is not out of reach. The TESLA study at DESY aims to develop these technologies and to build a $500 \mathrm{MV}$ prototype linac.

\section{The SBLC Approach}

In order to keep costs down, the SBLC collaboration is considering accelerating waveguides of $6 \mathrm{~m}$ in length. They will be used in pairs, with two waveguides powered by a single $150 \mathrm{MW}$ klystron. Special techniques ensure that the accelerating structure remains straight to better than $0.02 \mathrm{~mm}$. In order to avoid multibunch instabilities (125 bunches are to be accelerated in a $2 \mu$ s long pulse) all the beam-guiding elements are of the constant-gradient type. This provides for a variation in frequency of all deflecting modes along the waveguide. Also, different accelerating guides are built differently to further spread out deflecting mode frequencies. A system of higher (deflecting) mode absorbers on the accelerating waveguides damps deflecting modes while allowing wave-guide alignment for minimum deflecting mode amplitudes.

A particular problem of most linear colliders is the extremely small beam emittance, which makes the machines very susceptible to vibrations of the focusing quadrupoles. A solution may involve active vibration control systems which are being investigated by the SBLC collaboration.
The second approach that is being studied at DESY is a more-or-less conventional S-band accelerator under the acronym SBLC. S-band linear accelerators have been built for more than 45 years and a wealth of data exists on this type of machine. For a linear collider, however, these standard linacs have to be upgraded in two important directions: alignment and manufacturing tolerances must be improved by more than an order-of-magnitude, and special measures have to be taken to suppress any beam instabilities. Also, production costs for such a machine have to be lowered, and new technologies need to be developed with this in mind.
A plan of the $400 \mathrm{MeV}$ S-Band Test Facility at DESY. The prototype linac consists of 4 accelerating structures $(6 \mathrm{~m}$ in length) powered by two 150 MW klystrons. A special injection system will produce 125 tightly focused particle bunches in each $2 \mu \mathrm{s}$ pulse.

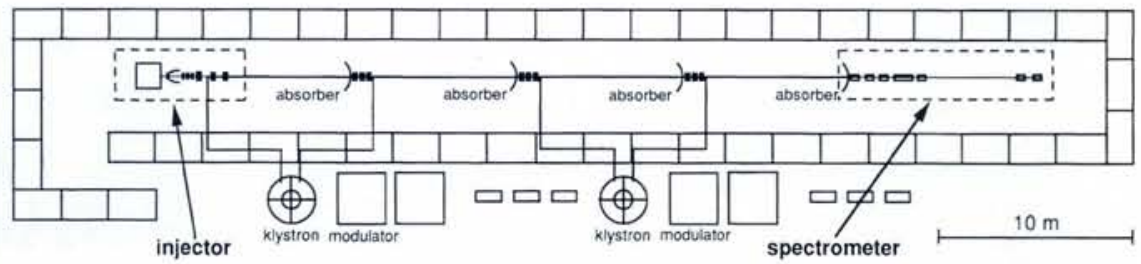

The aim of the on-going SBLC study is the construction of a $400 \mathrm{MeV}$ linac built in such a way that it would be suitable for a $30 \mathrm{~km}$ long linear collider. This prototype linac will not only allow the study and development of technical components such high-power, $150 \mathrm{MW}$ klystrons, high-power modulators, accelerating guides with high straightness and mode absorbers, quadrupole magnets with active vibration control, etc., but it will also allow the study of higher order mode excitation and its effect in deliberately misaligned accelerating guides. In this way it will be possible to verify the computer simulations being developed to study the effect of emittance preservation. All of the work mentioned is in progress and the SBLC prototype linac is expected to be complete in 1996.

\section{Co-operation Essential}

In recent decades, particle physicists have increasingly concentrated their efforts on a few large-scale projects. Groups of scientists from many countries are therefore collaborating in constructing new particle accelerators and detectors. In Europe, CERN and DESY provide outstanding resources for pursuing unresolved questions in particle physics.

The European Committee for Future Accelerators (ECFA) is successfully coordinating the particle-physics research programme by making recommendations for the construction of new facilities. In the past, ECFA unanimously endorsed the building of the world's largest electron-positron collider (LEP) and the Large Hadron Collider (LHC) at CERN, and the construction of the world's only hadron-electron collider (HERA) at DESY in Hamburg.

European co-operation in particle physics is embedded in combined efforts across the world, based on reciprocal utilization, free of charge, of the few large accelerators. A prime example of European cooperation in particle physics is provided by HERA in Hamburg. Planned since the end of the 1970s, approved in May 1984, and put into operation in 1991, HERA is now available to scientists from all over the world. When a decision about the HERA project was to be taken, DESY's funding agencies (with $90 \%$ of the money from the Ministry of Research and Technology in Bonn and 10\% from the Land of Hamburg) linked approval to the condition that nonGerman research organizations were willing to make contributions to the project. These contributions were successfully organized without special inter-governmental agreements and were coordinated by a stee- ring committee composed of members from all participating countries. The foreign partners provided major components and services for HERA and in turn profited from participation in the know-how. This mode of contributing to the construction of an accelerator became known as the "HERA model".

As an example, the Istituto Nazionale di Fisica Nucleare (INFN) in Italy declared early on its readiness to participate. As the Italian contribution to HERA, it would supply half of the 422 superconducting dipole magnets required for the proton ring, partly because large Italian firms were very interested in manufacturing this new type of magnet. After the initial development of the superconducting dipole magnets at DESY and the construction and successful test of a dozen bending magnets by German and Italian firms, series production was started in industry. The outcome of this close co-operation with industry was excellent. About 45 foreign institutes and 320 firms (almost all of them from Europe) were involved in the construction of the HERA accelerator complex. Many European enterprises were thereby able, in collaboration with DESY, to gain experience in very modern technologies, particularly in the field of superconductivity and associated areas.

Co-operation among the experimentalists is even more multi-national and far-ranging. In the case of the experiments $\mathrm{H} 1$ and ZEUS, where foreign financial involvement amounts to $60 \%$, it is hardly possible any longer to talk about international "participation" as they are truly international enterprises. A characteristic feature of such a co-operation in an experiment is the fact that the development and construction of individual detector components takes place under the sole technical and financial responsibility of the participating institutes.
Scientists from institutes in more than 20 countries around the world are carrying out research at HERA in trying to gain insight into the structure of matter. They will continue to be involved for many years in the analysis of data generated at HERA's' experiments. Scientific co-operation with institutes from the former eastern bloc will continue under this framework, which was successfully established long before the end of the east-west conflict.

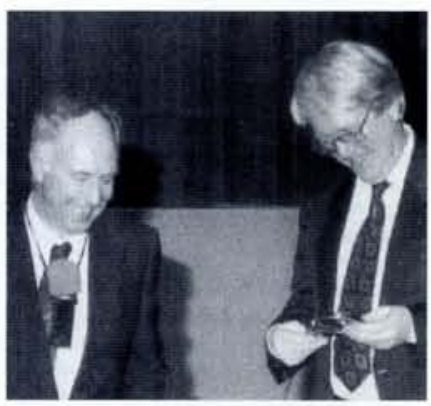

B.H. Wiik (on the right) receiving the symbolic DESY key from $V$. Soergel shortly after he took over as DESY's Director General last year. 